Bull. Mater. Sci., Vol. 6, No. 2, May 1984, pp. 273-281. (C) Printed in India.

\title{
On the mechanism of pairing in the BCs theory
}

\author{
C M SRIVASTAVA \\ Department of Physics and Advanced Centre for Research in Electronics, Indian Institute of \\ Technology, Bombay 400 076, India
}

\begin{abstract}
An explanation has been offered for the mechanism of cooper pairing in the BCS theory of superconductivity on the basis of the relativistic Darwin interaction. The theory leads to an expression for critical temperature which depends on a few atomic parameters. Calculated values of $T_{c}$ for elements and alloys are in good agreement with experiment.
\end{abstract}

Keywords. Cooper pairing: BCS theory; superconductivity; pairing interaction; critical temperature.

\section{Introduction}

The BCs (Bardeen et al 1957) theory of superconductivity is based on the pairing hypothesis of a simple model of electron gas with attractive interaction. This interaction is assumed to be mediated by phonons. Despite the striking success of the BCs theory in explaining the essential properties of superconductors, attempts to increase the size of the pairing interaction using BCS theory have not met with much success and we have continued basic materials research without much help from theory (Gamota 1981; Matthias 1976; Hein 1981). It has been suggested that the systematics of $T_{c}$ in transition series elements can be characterized by an atomic-like parameter (Hopfield 1969; Bennemann et al 1972; Collver and Hammond 1973). In this paper we propose to show that pairing occurs due to the Darwin term in the relativistic Hamiltonian. The theory accounts for the success of the reduced BCs Hamiltonian and yields an expression for the dependence of $T_{c}$ on atomic parameters which is in good quantitative agreement with experiment.

\section{The pairing interaction}

The reduced BCs Hamiltonian

$$
H_{\mathrm{red}}=\sum_{k} \varepsilon_{k} c_{k}^{+} c_{-k}+\sum_{k, k^{\prime}} V_{k k^{\prime}} c_{k^{\prime}}^{+} c_{-k^{\prime}}^{+} c_{-k} c_{k}
$$

contains only the two-body correlations and not the higher ones. Any term not included in $H_{\text {red }}$ breaks the pair correlation. The reason for this has not been very clear (Cooper 1962; Blatt 1964) since the beginning of the BCs theory. We show below that this correlation originates due to the Darwin interaction. 
It is known that mass-velocity and Darwin terms in the relativistic Hamiltonian have drastic effects on the energy band structure of solids (Johnson et al 1963). On account of the E.p form of the Darwin term, it is possible that in a two-electron system, the contribution from this repulsive term, under certain conditions, may become less due to the dynamical correlation in momentum and thereby leads to Cooper pair formation.

Consider a pair of electrons above a quiescent Fermi sphere with Darwin interaction. We assume that the charged particle interactions within the metal are coulombic so the electron-electron interaction is given by $e^{2} /\left|\mathbf{r}_{i}-\mathbf{r}_{j}\right|$ and the electron-ion interaction is given by $Z_{\text {eff }} e^{2} /\left|\mathbf{r}_{i}-\mathbf{R}_{j}\right|$ where $Z_{\text {eff }}$ is the effective ion valency seen by the electrons.

For a two electron system we take the product wave-function for a singlet spin state,

$$
\phi\left(\mathbf{k}_{1} ; \mathbf{k}_{2} ; \mathbf{x}_{1}, \mathbf{x}_{2}\right)=\frac{1}{\sqrt{2}}\left[\phi_{\mathbf{k}_{1}}\left(\mathbf{x}_{1}\right) \phi_{\mathbf{k}_{2}}\left(\mathbf{x}_{2}\right)+\phi_{\mathbf{k}_{2}}\left(\mathbf{x}_{1}\right) \phi_{\mathbf{k}_{1}}\left(\mathbf{x}_{2}\right)\right]
$$

where $\phi_{k_{i}}\left(\mathbf{x}_{j}\right)(i, j=1,2)$ are the one electron Bloch function for a perfectly periodic lattice

$$
\left\{-\frac{\hbar^{2}}{2 m} \nabla^{2}+U(\mathbf{x})\right\} \phi_{\mathbf{k}}(\mathbf{x})=\varepsilon_{\mathbf{k}} \phi_{\mathbf{k}}(\mathbf{x})
$$

The Darwin term in the relativistic Hamiltonian is (Bethe and Salpeter 1957)

$$
H_{D}=-\frac{i e \hbar}{(2 m c)^{2}}\left(\mathbf{E}_{1} \cdot \mathbf{p}_{1}+\mathbf{E}_{2} \cdot \mathbf{p}_{2}\right)
$$

where $\mathbf{E}_{1}$ and $\mathbf{E}_{2}$ are the electric field intensity at the electron positions $\mathbf{x}_{1}$ and $\mathbf{x}_{2}$ respectively.

We now use first-order perturbation theory to obtain the contribution of $H_{D}$ to the total energy. We assume that there is another state $\phi\left(\mathbf{k}_{1}^{\prime}, \mathbf{k}_{2}^{\prime}\right)$ which is degenerate with $\phi\left(\mathbf{k}_{1}, \mathbf{k}_{2}\right)$. For the singlet state it is sufficient to work with unsymmetrized product wavefunction. Using the momentum representation, the Bloch function is,

$$
\phi_{\mathbf{k}}(\mathbf{x})=\frac{1}{\sqrt{N \Omega}} \sum_{\mathbf{G}} f_{\mathbf{G}}(\mathbf{k}) \exp (i(\mathbf{G}+\mathbf{k}) \cdot \mathbf{x})
$$

where $\mathbf{G}$ is the reciprocal lattice vector, $f_{\mathbf{G}}(\mathbf{k})$ are $c$-numbers, $\Omega$ is the volume of the elcmentary cell and $N$ is the number of cells in the crystal. The diagonal matrix element of $H_{D}$ is,

$$
\begin{aligned}
H_{11}= & \left\langle\phi\left(\mathbf{k}_{1}, \mathbf{k}_{2}\right)\left|H_{D}\right| \phi\left(\mathbf{k}_{1}, \mathbf{k}_{2}\right)\right\rangle \\
= & -\frac{1}{2} \frac{i e \hbar}{(2 m c)^{2}}\left\langle\phi\left(\mathbf{k}_{1}, \mathbf{k}_{2}\right)\right|\left[\sum_{i=1,2} \mathbf{E}\left(\mathbf{x}_{i}\right) \cdot \mathbf{p}_{i}-\mathbf{p}_{i} \cdot \mathbf{E}\left(\mathbf{x}_{i}\right)\right] \\
& +\mathbf{E}\left(\mathbf{x}_{1}-\mathbf{x}_{2}\right) \cdot\left(\mathbf{p}_{1}-\mathbf{p}_{2}\right)-\left(\mathbf{p}_{1}-\mathbf{p}_{2}\right) \cdot \mathbf{E}\left(\mathbf{x}_{1}-\mathbf{x}_{2}\right)\left|\phi\left(\mathbf{k}_{1}, \mathbf{k}_{2}\right)\right\rangle .
\end{aligned}
$$

In (6) use has been made of the relation

$$
\left\langle\mathbf{E}_{1} \cdot \mathbf{p}_{1}+\mathbf{E}_{2} \cdot \mathbf{p}_{2}\right\rangle=-\left\langle\mathbf{p}_{1} \cdot \mathbf{E}_{1}+\mathbf{p}_{2} \cdot \mathbf{E}_{2}\right\rangle \text {, }
$$

which is valid as long as $\mathbf{E}_{1}\left(\mathbf{E}_{2}\right)$ is the special function- $\operatorname{grad}_{1} V\left(-\operatorname{grad}_{2} V\right)$ where $V$ is the sum of all potentials at $1(2)$.

Using Bloch function (5) we obtain,

$$
\left\langle\phi\left(\mathbf{k}_{1}, \mathbf{k}_{2}\right)\left|\sum_{i=1,2} \mathbf{E}\left(\mathbf{x}_{i}\right) \cdot \mathbf{p}_{i}-\mathbf{p}_{i} \cdot \mathbf{E}\left(\mathbf{x}_{i}\right)\right| \phi\left(\mathbf{k}_{1}, \mathbf{k}_{2}\right)\right\rangle
$$




$$
\begin{aligned}
= & \frac{Z_{\text {eff }}}{N \Omega} \sum_{\mathbf{G}_{1}, \mathbf{G}_{2}, \mathbf{G}_{3}, \mathbf{G}_{4}} f_{\mathbf{G}_{1}}^{*}\left(\mathbf{k}_{1}\right) f_{\mathbf{G}_{2}}^{*}\left(\mathbf{k}_{2}\right) f_{\mathbf{G}_{3}}\left(\mathbf{k}_{1}\right) f_{\mathbf{G}_{4}}\left(\mathbf{k}_{2}\right) \\
& \left\{\delta\left(\mathbf{G}_{2}, \mathbf{G}_{4}\right) \int \mathrm{d}^{3} \mathbf{x}_{1} \exp \left(i\left(\mathbf{G}_{3}-\mathbf{G}_{1}\right) \cdot \mathbf{x}_{1}\right) \frac{\mathbf{x}_{1} \cdot\left(\mathbf{G}_{3}-\mathbf{G}_{1}\right)}{\left|\mathbf{x}_{1}\right|^{3}}\right. \\
& \left.+\delta\left(\mathbf{G}_{1}, \mathbf{G}_{3}\right) \int \mathrm{d}^{3} \mathbf{x}_{2} \exp \left(i\left(\mathbf{G}_{4}-\mathbf{G}_{2}\right) \cdot \mathbf{x}_{2}\right) \frac{\mathbf{x}_{2} \cdot\left(\mathbf{G}_{4}-\mathbf{G}_{2}\right)}{\left|\mathbf{x}_{2}\right|^{3}}\right\} \\
= & \frac{4 \pi i}{N \Omega} Z_{\text {eff }} \text { e } \sum_{\mathbf{G}_{1}, \mathbf{G}_{2}, \mathbf{G}_{3}, \mathbf{G}_{4}} f_{\mathbf{G}_{1}}^{*}\left(\mathbf{k}_{1}\right) f_{\mathbf{G}_{2}}^{*}\left(\mathbf{k}_{2}\right) f_{\mathbf{G}_{3}}\left(\mathbf{k}_{1}\right) f_{\mathbf{G}_{4}}\left(\mathbf{k}_{2}\right) \\
& \left\{\delta\left(\mathbf{G}_{2}, \mathbf{G}_{4}\right)+\delta\left(\mathbf{G}_{1}, \mathbf{G}_{3}\right)\right\} .
\end{aligned}
$$

We obtain the contribution from the Coulomb repulsion term in (6) by changing to centre-of-mass and relative-motion coordinates

$$
\phi\left(\mathbf{k}_{1}, \mathbf{k}_{2}\right)=\frac{1}{N \Omega} \sum_{\mathbf{G}_{1}, \mathbf{G}_{2}} f_{\mathbf{G}_{1}}\left(\mathbf{k}_{1}\right) f_{\mathbf{G}_{2}}\left(\mathbf{k}_{2}\right) \exp (i \mathbf{k} \cdot \mathbf{X}) \exp (i \mathbf{k} \cdot \mathbf{x})
$$

where $X=\frac{1}{2}\left(\mathbf{x}_{1}+\mathbf{x}_{2}\right)$; $\quad \mathbf{X}=\mathbf{x}_{1}-\mathbf{x}_{2}$

$$
K=k_{1}+k_{2}+G_{1}+G_{2} ; \quad k=\frac{1}{2}\left(G_{1}+k_{1}-G_{2}-k_{2}\right) .
$$

Using (8) we obtain,

$$
\begin{aligned}
& \left\langle\phi\left(\mathbf{k}_{1}, \mathbf{k}_{2}\right)|\mathbf{E}(\mathbf{x}) \cdot \mathbf{p}-\mathbf{p} \cdot \mathbf{E}(\mathbf{x})| \phi\left(\mathbf{k}_{1}, \mathbf{k}_{2}\right)\right\rangle \\
& =-\frac{2 e \hbar}{N^{2} \Omega^{2}} \sum_{\mathbf{G}_{1}, \mathbf{G}_{2}, \mathbf{G}_{3}, \mathbf{G}_{4}} f_{\mathbf{G}_{1}}^{*}\left(\mathbf{k}_{1}\right) f_{\mathbf{G}_{2}}^{*}\left(\mathbf{k}_{2}\right) f_{\mathbf{G}_{3}}\left(\mathbf{k}_{1}\right) f_{\mathbf{G}_{4}}\left(\mathbf{k}_{2}\right) \\
& \int \mathrm{d}^{3} \mathbf{X} \exp \left(i\left(\mathbf{K}-\mathbf{K}^{\prime}\right) \cdot \mathbf{X}\right) \int \mathrm{d}^{3} \mathbf{x} \exp \left(i\left(\mathbf{k}-\mathbf{k}^{\prime}\right) \cdot \mathbf{x}\right) \frac{\mathbf{x} \cdot\left(\mathbf{k}-\mathbf{k}^{\prime}\right)}{|\mathbf{x}|^{3}} \\
& =-\frac{2}{N \Omega}(4 \pi i) e \hbar \sum_{\mathbf{G}_{1}, \mathbf{G}_{2}, \mathbf{G}_{3}, \mathbf{G}_{4}} f_{\mathbf{G}_{1}}^{*}\left(\mathbf{k}_{1}\right) f_{\mathbf{G}_{2}}^{*}\left(\mathbf{k}_{2}\right) f_{\mathbf{G}_{3}}\left(\mathbf{k}_{1}\right) f_{\mathbf{G}_{4}}\left(\mathbf{k}_{2}\right) \delta\left(\mathbf{K}, \mathbf{K}^{\prime}\right)
\end{aligned}
$$

where $\mathbf{K}=\mathbf{k}_{1}+\mathbf{k}_{2}+\mathbf{G}_{3}+\mathbf{G}_{4} \quad \mathbf{K}^{\prime}=\mathbf{k}_{1}+\mathbf{k}_{2}+\mathbf{G}_{1}+\mathbf{G}_{2}$.

From (6), (7) and (8)

$$
\begin{aligned}
H_{11}= & \frac{\pi}{2 N \Omega}\left(\frac{e \hbar}{m c}\right)^{2}\left[\sum_{\mathbf{G}_{1}, \mathbf{G}_{2}, \mathbf{G}_{3}, \mathbf{G}_{4}} f_{\mathbf{G}_{1}}^{*}\left(\mathbf{k}_{1}\right) f_{\mathbf{G}_{2}}^{*}\left(\mathbf{k}_{2}\right) f_{\mathbf{G}_{3}}\left(\mathbf{k}_{1}\right) f_{\mathbf{G}_{4}}\left(\mathbf{k}_{2}\right)\right. \\
& \left\{Z_{\mathrm{eff}} \delta\left(\mathbf{G}_{2}, \mathbf{G}_{4}\right)+Z_{\mathrm{eff}} \delta\left(\mathbf{G}_{1}, \mathbf{G}_{3}\right)-2 \delta\left(\mathbf{G}_{1}+\mathbf{G}_{2}, \mathbf{G}_{3}+\mathbf{G}_{4}\right)\right\}
\end{aligned}
$$

Due to the normalisation of $\phi_{\mathbf{k}_{2}}\left(\mathbf{x}_{2}\right)$, the summation over $G_{2}, G_{4}$ in the first term yields 1 . The other summation over $\mathbf{G}_{1}$ and $\mathbf{G}_{3}$ gives the expectation value of the Dirac delta function $\left\langle\delta^{3}\left(\mathbf{x}_{1}\right)\right\rangle$. Similarly, the second term leads to $\left\langle\delta^{3}\left(\mathbf{x}_{2}\right)\right\rangle$ and the electronelectron term gives $\left\langle\delta^{3}\left(\mathbf{x}_{1}-\mathbf{x}_{2}\right)\right\rangle$. So finally we obtain,

$$
H_{11}=\pi\left(\frac{e \hbar}{m c}\right)^{2}\left\{Z_{\text {eff }}\left\langle\delta^{3}\left(\mathbf{x}_{1}\right)\right\rangle-\left\langle\delta^{3}\left(\mathbf{x}_{1}-\mathbf{x}_{2}\right)\right\rangle\right\}
$$

where the expectation value of the Dirac delta function is taken to be the same for the states $\mathbf{k}_{1}$ and $\mathbf{k}_{2}$. In that case $H_{11}=H_{22}$. 
For the off-diagonal matrix element, we obtain

$$
\begin{aligned}
H_{12}= & \left\langle\phi\left(\mathbf{k}_{1}^{\prime}, \mathbf{k}_{2}^{\prime}\right)\left|H_{D}\right| \phi\left(\mathbf{k}_{1}, \mathbf{k}_{2}\right)\right\rangle \\
= & \frac{\pi}{2 N \Omega}\left(\frac{e \hbar}{m c}\right)^{2}\left[\sum_{\mathbf{G}_{1}, \mathbf{G}_{2}, \mathbf{G}_{3}, \mathbf{G}_{4}} f_{\mathbf{G}_{1}}^{*}\left(\mathbf{k}_{1}^{\prime}\right) f_{\mathbf{G}_{2}}^{*}\left(\mathbf{k}_{2}^{\prime}\right) f_{\mathbf{G}_{3}}\left(\mathbf{k}_{1}\right) f_{\mathbf{G}_{4}}\left(\mathbf{k}_{2}\right)\right. \\
& \left\{Z_{\text {eff }} \delta\left(\mathbf{G}_{4}+\mathbf{k}_{2}, \mathbf{G}_{2}+\mathbf{k}_{2}^{\prime}\right)+Z_{\mathrm{eff}} \delta\left(\mathbf{G}_{3}+\mathbf{k}_{1}, \mathbf{G}_{1}+\mathbf{k}_{1}^{\prime}\right)\right. \\
& \left.\left.-2 \delta\left(\mathbf{G}_{3}+\mathbf{G}_{4}+\mathbf{k}_{1}+\mathbf{k}_{2}, \mathbf{G}_{1}+\mathbf{G}_{2}+\mathbf{k}_{1}^{\prime}+\mathbf{k}_{2}^{\prime}\right)\right\}\right]
\end{aligned}
$$

If the states $\left(\mathbf{k}_{1}, \mathbf{k}_{2}\right)$ and $\left(\mathbf{k}_{1}^{\prime}, \mathbf{k}_{2}^{\prime}\right)$ are uncorrelated, the delta functions in (12) vanish so $H_{12}$ is zero. The net contribution to energy from the Darwin interaction is then given by (11) which is positive because

$$
\left(\left\langle\delta^{3}\left(\mathbf{x}_{1}\right)\right\rangle \gg\left\langle\delta^{3}\left(\mathbf{x}_{1}-\mathbf{x}_{2}\right)\right\rangle\right) .
$$

Let us now examine the special case when

$$
\left.\begin{array}{rl}
\mathbf{k}_{1}=-\mathbf{k}_{2}=\mathbf{k} ; & \mathbf{k}_{1}^{\prime}=-\mathbf{k}_{2}^{\prime}=\mathbf{k}^{\prime} . \\
\mathbf{G}_{2}=\mathbf{G}-\mathbf{G}_{1} ; & \mathbf{G}_{3}=\mathbf{G}-\mathbf{G}_{4} .
\end{array}\right\}
$$

In this case $H_{12}$ has a non-vanishing value,

$$
\begin{aligned}
H_{12}= & \frac{\pi}{2 N \Omega}(e \hbar / m c)^{2} \sum_{\mathbf{G}_{1}, \mathbf{G}_{3}, \mathbf{G}} f_{\mathbf{G}_{1}}^{*}\left(\mathbf{k}^{\prime}\right) f_{\mathbf{G}-\mathbf{G}_{1}}^{*}\left(-\mathbf{k}^{\prime}\right) f_{\mathbf{G}_{3}}(\mathbf{k}) f_{\mathbf{G}-\mathbf{G}_{3}}(-\mathbf{k}) \\
& \left\{Z_{\mathrm{eff}} \delta\left(\mathbf{G}-\mathbf{G}_{3}-\mathbf{k}, \mathbf{G}-\mathbf{G}_{1}-\mathbf{k}^{\prime}\right)+Z_{\mathrm{eff}} \delta\left(\mathbf{G}_{1}+\mathbf{k}^{\prime}, \mathbf{G}_{3}+\mathbf{k}\right)-2\right\}
\end{aligned}
$$

The first two terms give nonvanishing values for $\mathbf{k}^{\prime}-\mathbf{k}=\mathbf{G}_{3}-\mathbf{G}_{1}=\mathbf{G}_{\mathbf{0}}$ where $\mathbf{G}_{0}$ is a reciprocal lattice vector. The degeneracy is now removed and there are two states having energy,

$$
E_{1,2}=H_{11} \pm H_{12}
$$

Since s-like function has spatial charge distribution which over a large volume is spherically symmetric, the fourier coefficients in the terms having the same phase factor should be the same:

$$
\begin{aligned}
\phi_{\mathbf{k}}(\mathbf{x}) & =\frac{1}{\sqrt{N \Omega}} \sum_{\mathbf{G}} f_{\mathbf{G}}(\mathbf{k}) \exp (i(\mathbf{G}+\mathbf{k}) \cdot \mathbf{x}), \\
\phi_{\mathbf{k}^{\prime}}\left(\mathbf{x}^{\prime}\right) & =\frac{1}{\sqrt{N \Omega}} \sum_{G^{\prime}} f_{\mathbf{G}^{\prime}}\left(\mathbf{k}^{\prime}\right) \exp \left(i\left(\mathbf{G}^{\prime}+\mathbf{k}^{\prime}\right) \cdot \mathbf{x},\right. \\
\text { then } f_{\mathbf{G}}(\mathbf{k}) & =f_{\mathbf{G}^{\prime}}\left(\mathbf{k}^{\prime}\right), \text { provided } \mathbf{G}+\mathbf{k}=\mathbf{G}^{\prime}+\mathbf{k}^{\prime} .
\end{aligned}
$$

In this case $H_{12}$ is equal to $H_{11}$ and the net energy in the phase coherent state defined by (13) is lower than in the uncorrelated phase by $H_{11}=H_{12}$. In this state the Cooper pairs are continuously scattered so that these suffer Bragg reflections as frequently as possible. With BCs we assume that only the electrons in a thin shell of width $\pm \hbar w_{D}$ about the Fermi surface are effective in producing the superconducting phase. The vanishing 
of the repulsive Darwin interaction would ensure that $V_{k k^{\prime}}<0$ so the reduced BCs Hamiltonian of (1) describes the coherent state completely.

\section{The critical temperature}

The BCs (Bardeen et al 1957) solution of $H_{\text {red }}$ yields the energy required to break the Cooper pair as $2 \Delta_{0}$ where $\Delta_{0}$ is the energy gap parameter. BCs have also established the relation between $\Delta_{0}$ and the critical temperature, $T_{c}$. Using these we have,

$$
2 \Delta_{0}=H_{11}=H_{12}=3.5 k_{B} T_{c} .
$$

Substituting from (11) in (16),

$$
k_{B} T_{c}=\frac{\pi}{3 \cdot 5}(e h / m c)^{2}\left\{Z_{\mathrm{eff}}\left\langle\delta^{3}\left(\mathbf{x}_{1}\right)\right\rangle-\left\langle\delta^{3}\left(\mathbf{x}_{1}-\mathbf{x}_{2}\right)\right\rangle\right\}
$$

It is expected that $\left\langle\delta^{\mathbf{3}}\left(\mathbf{x}_{1}\right)\right\rangle$ would be larger than $\left\langle\delta^{\mathbf{3}}\left(\mathbf{x}_{1}-\mathbf{x}_{2}\right)\right\rangle$. For He the strength of the electron-ion $(e-i)$ and electron-electron $(e-e)$ terms are $132.95 \mathrm{~cm}^{-1}$ and $3.92 \mathrm{~cm}^{-1}$ respectively (Bethe and Salpeter 1957). Further it is found that for He the spin magnetic dipole interaction (Fermi contact term) leads also to a delta function interaction, $\left\langle\delta^{3}\left(\mathbf{x}_{1}-\mathbf{x}_{2}\right)\right\rangle$, which is positive and twice the magnitude of the $e-e$ Darwin term. Since $(e-e)$ term is expected to be less than $5 \%$ of the $(e-i)$ term we may write,

$$
k_{B} T_{c} \simeq \frac{\pi}{3.5}(e \hbar / m c)^{2} Z_{\mathrm{eff}}\left\langle\psi_{F}^{2}(0)\right\rangle_{\mathrm{av}}
$$

where the probability density at the nucleus $\left\langle\psi_{F}^{2}(0)\right\rangle_{\text {av }}$ is evaluated over the $s$-like electrons at the Fermi surface.

For hydrogen-like wavefunctions we may approximately take,

$$
\left\langle\psi_{F}^{2}(0)\right\rangle=Z_{\mathrm{eff}}^{3} / \pi a_{H}^{3} n_{\mathrm{eff}}^{3},
$$

where $Z_{\text {eff }}$ is the effective nuclear charge experienced by the valence $s$-electrons, $n_{\text {eff }}$ is the effective principal quantum number and $a_{H}$ is the Böhr radius. Substituting from (18) in (17a) we have,

$$
T_{c}=0.285\left(m c^{2} \alpha^{4} Z_{\text {eff }}^{4}\right) /\left(k_{B} n_{\text {eff }}^{3}\right),
$$

where $\alpha$ is the fine structure constant. We may take the Slater orbital values of $n_{\mathrm{eff}}$ as these give reasonably good values for free atoms. For $Z_{\text {eff }}$ it is found that the experimental data fits the relation,

$$
Z_{\mathrm{eff}}=a\left(r_{s}-b\right)
$$

where $r_{s}$ is the mean electronic separation parameter and $a$ and $b$ are constants.

\subsection{Localised versus itinerant states}

To determine $r_{s}$ for simple metals with fixed formal valencies we assume that $Z_{0}$ electrons fill each atomic sphere, where $Z_{0}$ is the valency. For metals with variable valencies, complete ionization is often not achieved (Hume-Rothery et al 1962) and we need to assign an effective degree of ionization $Z_{0}^{*} \leqslant Z_{0}$. The values of $Z_{0}^{*}$ determined from the data on $T_{c}$ are given in table 1 . These values are in good agreement with the 
Table 1. Atomic parameters related to $T_{c}$

Effective principal quantum number (Slater orbital)

$\begin{array}{llllllll}n \rightarrow & 1 & 2 & 3 & 4 & 5 & 6 & 7 \\ n_{\text {eff }} \rightarrow & 1 & 2 & 3 & 3 \cdot 7 & 4 \cdot 0 & 4 \cdot 2 & 4 \cdot 4\end{array}$

\begin{tabular}{|c|c|c|c|c|c|c|}
\hline Element & $n$ & $Z_{0}$ & $Z_{0}^{*}$ & $r_{3}$ & $\begin{array}{c}\text { Metallic } \\
Z_{\mathrm{eff}}=a\left(r_{\mathrm{s}}-b\right)\end{array}$ & $\begin{array}{c}\text { Atomic } \\
Z_{\text {eff }}(\text { Slater rule })\end{array}$ \\
\hline \multicolumn{7}{|l|}{ Class 1} \\
\hline Al & 3 & 3 & 3 & 2.06 & 1.55 & $2 \cdot 30$ \\
\hline $\mathrm{Zn}_{\mathbf{n}}$ & 4 & 2 & 2 & $2 \cdot 30$ & $2 \cdot 15$ & $1 \cdot 70$ \\
\hline $\mathbf{G a}$ & 4 & 3 & 3 & $2 \cdot 18$ & 1.86 & $2 \cdot 30$ \\
\hline $\mathrm{Cd}$ & 5 & 2 & 2 & $2 \cdot 59$ & $2 \cdot 70$ & $1 \cdot 70$ \\
\hline In & 5 & 3 & $2 \cdot 5$ & 2.52 & $2 \cdot 56$ & $2 \cdot 30$ \\
\hline $\mathrm{Sn}$ & 5 & 4 & $2 \cdot 5$ & 2.55 & $2 \cdot 62$ & 2.95 \\
\hline $\mathrm{La}$ & 6 & 3 & 3 & $2 \cdot 70$ & 2.92 & $1 \cdot 70$ \\
\hline $\mathbf{H g}$ & 6 & 2 & 2 & 2.66 & $2 \cdot 85$ & $1 \cdot 70$ \\
\hline $\mathrm{Tl}$ & 6 & 3 & 3 & $2 \cdot 48$ & 2.68 & $2 \cdot 30$ \\
\hline $\mathrm{Pb}$ & 6 & 4 & 2 & $2 \cdot 86$ & $3 \cdot 39$ & $2 \cdot 95$ \\
\hline $\mathrm{Ti}$ & 4 & 4 & 4 & 1.93 & $1 \cdot 34$ & 1.95 \\
\hline $\mathbf{Z r}$ & 5 & 4 & 4 & $2 \cdot 11$ & $1 \cdot 71$ & 1.95 \\
\hline Hf & 6 & 4 & 4 & 2.08 & 1.66 & 1.90 \\
\hline Th & 7 & 4 & 4 & $2 \cdot 36$ & $2 \cdot 25$ & 1.95 \\
\hline $\mathbf{U}$ & 7 & 3 & 3 & $2 \cdot 23$ & $2 \cdot 35$ & 1.80 \\
\hline \multicolumn{7}{|l|}{ Class 2} \\
\hline V & 4 & 5 & $2 \cdot 5$ & 2.03 & $2 \cdot 81$ & $2 \cdot 10$ \\
\hline $\mathrm{Nb}$ & 5 & 5 & 3 & $2 \cdot 13$ & 3.09 & 1.60 \\
\hline Mo & 5 & 6 & 4 & 1.85 & 1.83 & $1 \cdot 75$ \\
\hline $\mathrm{Tc}$ & 5 & 7 & 3 & $2 \cdot 12$ & $3 \cdot 23$ & $2 \cdot 40$ \\
\hline $\mathbf{R u}$ & 5 & 6 & 4 & 1.77 & 1.43 & $2 \cdot 05$ \\
\hline $\mathrm{Ta}$ & 6 & 5 & $3 \cdot 5$ & 2.03 & 2.81 & $2 \cdot 10$ \\
\hline $\mathbf{w}$ & 6 & 6 & 6 & 1.62 & 0.64 & $2 \cdot 25$ \\
\hline $\operatorname{Re}$ & 6 & 7 & 3.5 & 1.89 & 2.06 & $2 \cdot 40$ \\
\hline Os & 6 & 6 & 3.0 & 1.89 & 2.06 & 3.55 \\
\hline Ir & 6 & 6 & 4.0 & 1.79 & 1.65 & $2 \cdot 70$ \\
\hline
\end{tabular}

$n=$ Principal quantum number, $n_{\mathrm{eff}}=$ effective principal quantum number, $Z_{0}=$ valency of the element, $Z_{0}^{*}=$ degree of ionization in the metallic state, $Z_{\text {eff }}=$ effective nuclear charge seen by the electrons.

values suggested by others. For example, according to Pauling (1949) the average valency per atom in white tin is 2.5 . Similarly Hume-Rothery et al (1962) reported that the atoms in $\mathrm{In}, \mathrm{Pb}$ and $\mathrm{Ti}$ are incompletely ionized which supports our conclusions.

The constants $a$ and $b$ in (20) have been determined from the data on $T_{c}$. Analysis shows that these constants are different for $Z_{0} \leqslant 4$ and $Z_{0} \geqslant 5$. Denoting the former as Class 1 and the latter as Class 2 , we have obtained the values of $a$ and $b$ which are given in table 2.

Table 2. Values of $a$ and $b$ in eq. (20) for metals of classes 1 and 2 .

\begin{tabular}{lll}
\hline Class & \multicolumn{1}{c}{$a$} & $b$ \\
\hline 1 & 2.07 & 1.28 \\
2 & 5.29 & 1.50 \\
\hline
\end{tabular}


Table 3. $T_{\mathrm{c}}$ for elements

\begin{tabular}{|c|c|c|c|c|c|}
\hline \multirow{2}{*}{$\begin{array}{l}\text { Element } \\
\text { class 1 }\end{array}$} & \multicolumn{2}{|c|}{$T_{c}(K)$} & \multirow{2}{*}{$\begin{array}{l}\text { Element } \\
\text { class } 2\end{array}$} & \multicolumn{2}{|c|}{$T_{\mathrm{f}}(K)$} \\
\hline & Calc. & Obs. & & Calc. & Obs. \\
\hline Al & $1 \cdot 21$ & 1.20 & V & 5.94 & $5 \cdot 30$ \\
\hline $\mathrm{Zn}$ & 1.88 & 0.88 & $\mathrm{Nb}$ & $9 \cdot 36$ & 9.50 \\
\hline $\mathrm{Ga}$ & 1.14 & $1 \cdot 10$ & Mo & 0.88 & 0.92 \\
\hline $\mathrm{Cd}$ & 4.06 & 0.53 & $\mathrm{Tc}$ & 8.78 & 8.82 \\
\hline In & 3.26 & $3 \cdot 4$ & $\mathbf{R u}$ & 0.32 & 0.49 \\
\hline Sn & 3.58 & 3.72 & $\mathrm{Ta}$ & 4.06 & 4.48 \\
\hline La & 4.83 & 4.90 & W & 0.01 & 0.01 \\
\hline $\mathrm{Hg}$ & $4 \cdot 32$ & $4 \cdot 15$ & $\operatorname{Re}$ & $1 \cdot 17$ & 1.70 \\
\hline $\mathrm{T}]$ & $2 \cdot 47$ & $2 \cdot 39$ & Os & $1 \cdot 17$ & 0.66 \\
\hline$\ddot{P b}$ & $7 \cdot 42$ & $7 \cdot 19$ & Ir & $0-37$ & $0 \cdot 11$ \\
\hline $\mathrm{Ti}$ & $0-31$ & 0.39 & & & \\
\hline $\mathrm{Zr}$ & 0.66 & 0.52 & & & \\
\hline Hf & 0.50 & 0.09 & & & \\
\hline Th & 1.41 & 1.37 & & & \\
\hline $\mathrm{U}$ & 0.87 & 0.80 & & & \\
\hline
\end{tabular}

\section{$3.2 T_{c}$ for elements}

In table 1 parameters relating to $T_{c}$ are given for elements in Class 1 and 2 . The values of $Z_{\text {eff }}$ obtained from (19) are compared with those obtained using slater rules for free atoms.

The values of $T_{c}$ for elements are given in table 3. The experimental values are from Roberts (1964). These are in good agreement with theory except for $\mathrm{Zn}, \mathrm{Cd}$ and $\mathrm{Hf}$. The discrepancy in $\mathrm{Zn}$ and $\mathrm{Cd}$ may be due to the large departures in axial ratio $c / a$ from the ideal value 1.633 for hcp structures $(\mathrm{Zn}: 1.86, \mathrm{Cd}: 1.89)$. This makes the six in-plane $n-n$ closer to the central atom compared to the six out of plane $n-n$. Thus the values of $r_{s}$ are not properly assessed. In $\mathrm{Hf}$ again $Z_{\text {eff }}$ may not be given by the simple relation (20). The theory gives satisfactory values for $T_{c}$ of class 2 elements.

\section{$3.3 T_{c}$ for alloys}

We find that (19) and (20) also hold for many binary systems. The following rules based on the law of averages are generally applicable to these systems.

(i) A binary alloy $A_{x} B_{y}$ behaves as class 1 or 2 depending on the weighted mean value of the valence electrons per atom,

$$
\bar{Z}_{0}=\frac{x Z_{0}(A)+y Z_{0}(B)}{x+y}
$$

where $Z_{0}(A)$ and $Z_{0}(B)$ denote the valency of atoms $A$ and $B$ respectively. For $\bar{Z}_{0}<4$, the alloy behaves as Class 1 , while for $\bar{Z}_{0}>5$ it behaves as Class 2 . For $4 \leqslant \bar{Z}_{0} \leqslant 5$, the alloy may behave as either type depending on the nature of the constituent atoms.

(ii) The weighted mean $\bar{r}_{s}$ for a binary alloy is given by

$$
\bar{r}_{s}=\frac{x r_{A}+y r_{B}}{x+y}
$$


Table 4. $T_{c}$ for some binary compounds, $A_{x} B_{y}$

\begin{tabular}{|c|c|c|c|c|c|c|c|c|}
\hline \multirow{2}{*}{$\begin{array}{l}\text { Compound } \\
A_{x} B_{y}\end{array}$} & \multirow[b]{2}{*}{$\bar{Z}_{0}$} & \multirow[b]{2}{*}{ Class } & \multicolumn{2}{|c|}{$Z_{0}^{*}$} & \multirow[b]{2}{*}{$n$} & \multirow[b]{2}{*}{$\bar{r}_{s}$} & \multicolumn{2}{|c|}{$T_{c}(K)$} \\
\hline & & & $A$ & $B$ & & & Calc. & Obs. \\
\hline Al $\mathbf{M O}_{3}$ & $5 \cdot 25$ & 2 & 3 & 5 & 3 & 1.73 & $0-46$ & 0.58 \\
\hline $\mathrm{Al} \mathrm{Zr}_{3}$ & 3.75 & 1 & 3 & 4 & 3 & $2 \cdot 12$ & 1.66 & 0.73 \\
\hline $\mathrm{Ga} \mathrm{MO}_{3}$ & $5 \cdot 25$ & 2 & 3 & 5 & 4 & 1.84 & 1.05 & 0.76 \\
\hline $\mathrm{Ga} \mathrm{Nb}_{3}$ & $4 \cdot 50$ & 2 & 3 & 3 & 4 & $2 \cdot 15$ & $13 \cdot 27$ & 12.50 \\
\hline $\mathrm{In}_{2} \mathrm{Ag}$ & $2 \cdot 4$ & 1 & 3 & 2 & 5 & 2.41 & $2 \cdot 30$ & $2 \cdot 30$ \\
\hline In $\mathrm{Pb}$ & 2.5 & 1 & 2.5 & 2 & 5 & 2.69 & 6.78 & 6.65 \\
\hline In $\mathrm{Nb}_{3}$ & $4 \cdot 5$ & 2 & 3 & $3 \cdot 5$ & 5 & $2 \cdot 13$ & 11.07 & $9 \cdot 2$ \\
\hline $\mathrm{Tl} \mathrm{Au}$ & $3 \cdot 0$ & 1 & 3 & 2 & 6 & 2.44 & $2 \cdot 12$ & 1.92 \\
\hline $\mathrm{Sn} \mathrm{Ta}_{3}$ & 4.75 & 2 & 4 & 3.5 & 5 & 2.05 & $5 \cdot 50$ & 6.00 \\
\hline $\mathrm{Pb}_{2} \mathrm{Pd}$ & $4 \cdot 00$ & 1 & 2 & 4 & 5 & 2.54 & $3 \cdot 48$ & 2.95 \\
\hline $\mathrm{Sb} \mathrm{V}_{3}$ & 5.00 & 1 & 3 & 3 & 4 & $2 \cdot 10$ & $0-80$ & $0-80$ \\
\hline $\mathrm{Sb} \mathrm{Ti} i_{3}$ & 4.25 & 2 & 3 & 4 & 4 & 2.08 & $8 \cdot 30$ & $5 \cdot 80$ \\
\hline $\mathrm{Bi}_{2} \mathbf{P d}$ & $4 \cdot 70$ & 1 & 3 & 4 & 5 & $2 \cdot 39$ & $2 \cdot 10$ & $1 \cdot 70$ \\
\hline $\mathrm{Ti}_{0.8} \mathrm{~V}_{0.2}$ & $4 \cdot 20$ & 2 & 4 & 2.5 & 4 & 1.93 & 0.30 & 0.39 \\
\hline $\mathrm{Ti}_{0.25} \mathrm{~V}_{0.75}$ & 4.75 & 2 & 3.0 & 2.5 & 4 & 2.05 & 6.81 & $7 \cdot 16$ \\
\hline$V_{0.99} C_{0.01}$ & $5 \cdot 0$ & 2 & $3 \cdot 0$ & 3.0 & 4 & 1.95 & 3.06 & 4.8 \\
\hline $\mathrm{V}_{0.9} \mathrm{Cr}_{0.1}$ & $5 \cdot 1$ & 2 & $4 \cdot 0$ & $3 \cdot 0$ & 4 & $1 \cdot 78$ & 06 & 0.4 \\
\hline $\mathrm{Nb}_{0.4} \mathrm{Zr}_{0.6}$ & $4 \cdot 2$ & 2 & 3.0 & 4.0 & 5 & $2 \cdot 12$ & 8.83 & 8.80 \\
\hline $\mathrm{Nb}_{0.9} \mathrm{Zr}_{0 \cdot 1}$ & 4.9 & 2 & 3.0 & 3.0 & 5 & $2 \cdot 15$ & 10.52 & $10 \cdot 50$ \\
\hline
\end{tabular}

$\bar{Z}_{0}$ is the average valency obtained using (21) of the text, $Z_{0}^{*}$ is the degree of ionization, $n$ is the effective principal quantum number, $\bar{r}_{s}$ is the average value of $r_{s}$ obtained using (22).

where $r_{A}$ and $r_{B}$ are the $r_{S}$ values of pure metals $A$ and $B$.

(iii) The degree of ionization, $Z_{0}^{*}$ of the constituent atoms in the alloy may sometimes not be the same as in the metal and may vary between the formal valencies of elements depending on the relative values of the electronegativity of the constituents.

(iv) If the elements $A$ and $B$ in the alloy $A_{x} B_{y}$ belong to the same period, the effective principal quantum number is the same as for the constituents. When these do not belong to the same period, for $x \gg y(y \gg x), n_{\mathrm{eff}}$ is that for the atom $A(B)$. In the intermediate case, it is lower of the two values, $n_{\mathrm{eff}}(A)$ and $n_{\mathrm{eff}}(B)$.

A few examples of the calculated $T_{c}$ for the alloys of sp-metals and transition metals are given in table 4 . In most cases the theory gives satisfactory results.

\section{Criterion for superconductivity}

The regularity in the $T_{c}$ of the elements of the periodic table was first emphasized by Matthias (1957). This is now found to hold also for compounds and alloys. Some of the important regularities are

(i) Superconductivity is observed only in metals and is found in elements for which $2 \leqslant Z_{0} \leqslant 8$. The same generally holds for compounds also.

On our model superconductivity arises when the Fermi surface is cut by the Brillouin zone boundaries. The electrons in the Cooper pair state $(k \uparrow,-k \downarrow)$ move continuously 
through states on the constant energy surface until they reach a Bragg-reflection plane. At this point they get diffracted and the wavenumbers are changed by a reciprocal lattice vector $\mathbf{G}_{0} ; \mathbf{k}^{\prime}=\mathbf{k}+\mathbf{G}_{\mathbf{0}},-\mathbf{k}^{\prime}=-\mathbf{k}-\mathbf{G}_{0}$. For the contribution to energy from the Darwin interaction to vanish the Bragg-reflections should occur frequently. This would require that Fermi surface should be cut by as many Brillouin Zone (Bz) boundaries as possible to increase $T_{c^{*}}$. This is the reason that metals and alloys with $Z \leqslant 2$ do not become superconductors. For $Z=1$ the Fermi sphere lies well within the first Bz. For $Z$ $=2$ the sphere barely touches the $\mathrm{Bz}$. These metals therefore do not superconduct.

(ii) The variation of $T_{c}$ with e/a i.e. the number of valence electrons per atom is different for the nontransition and transition-elements. In the former it is a monotonic increase of $T_{c}$ with $e / a$. In the latter these are maxima at $e / a=3,5$ and 7 .

Since the number of electron pairs formed in the superconducting phase will increase with the number of electrons available at the Fermi surface, $T_{c}$ should increase with increase in the density of state at the Fermi surface, $N(0)$. This qualitatively explains the reason for high $T_{c}$ for odd values of $e / a$ in transition metals.

(iii) Certain regularities are connected to crystal structure. A-15 and Cheveral phase compounds [Hein 1981] show high $T_{c}$. This is due to the complex shape of the Brillouin Zone boundaries in these structures which increases the number of intersections with the Fermi Surface. There are also no superconductors which do not possess a centre of inversion. This follows since the two electrons in the Cooper pair state are needed to be scattered by $\mathbf{G}_{0}$ and $-\mathbf{G}_{0}$ reciprocal lattice vectors.

\section{Conclusion}

We have outlined a new physical situation under which the Bcs reduced Hamiltonian is shown to be applicable for the superconducting systems. The critical temperature derived from the model agrees well for metals and alloys.

\section{References}

Bardeen J, Cooper L N and Schrieffer J R 1957 Phys. Rev. 1081175

Bennemann K H and Garland J W 1972 in Superconductivity in d-and f-band metals (ed.) D H Douglass (New York: Am. Inst. Phys.) pp. 103

Bethe H A and Salpeter E E 1957 in Handbuch der Physik (Berlin: Springer-Verlag) Vol. 35/1, p. 88

Blatt J M 1964 Theory of superconductivity (New York: Academic Press) pp. 181

Collver M M and Hammond R H 1973 Phys. Rev. Lett. 3092

Cooper L N 1962 Phys. Rev. Lett. 8367

Gamota G 1981 IEEE Trans. Mag. MAG-17 19

Hein R A 1981 IEEE Trans. Mag. MAG-17 p. 607

Hopfield J 1969 Phys. Rev. 186443

Hume-Rothery W and Rayner G V 1962 The structure of metals and alloys (London: Institution of Metals) p. 55

Johnson L E, Conklin J B and Pratt G W Jr 1963 Phys. Rev. Lett. 11538

Matthias B T 1976 in Superconductivity in d-and f-band metals (ed.) D H Douglass (New York: Plenum) p. 635

Pauling L 1949 Proc. R. Soc. (London) A196 343

Roberts B W 1964 in Progress in cryogenics (ed.) K Mendelssolın (London: Heywood) Vol. 4, p. 159. 